\title{
Air-holes radius change effects and structure transitions in the linear photonic crystal nanocavities
}

\author{
Ahmadreza Daraei* , Foroogh Khozeymeh Sarbisheh \\ Dept of Physics, Faculty of Science, University of Sistan and Baluchestan, Zahedan, Iran
}

Email address:

Daraei@phys.usb.ac.ir(A. Daraei)

\section{To cite this article:}

Ahmadreza Daraei, Foroogh Khozeymeh Sarbisheh. Air-Holes Radius Change Effects and Structure Transitions in the Linear Photonic Crystal Nanocavities. American Journal of Optics and Photonics. Vol. 1, No. 3, 2013, pp. 11-16. doi: 10.11648/j.ajop.20130103.11

\begin{abstract}
The strong localization of electromagnetic modes in high-Q/ $\mathrm{V}_{\mathrm{m}}$ (quality factor/modal volume) photonic crystal $(\mathrm{PhC})$ nanocavities (NCs) makes them strong candidates for enhanced optical nonlinear effects, such as cavity quantum electrodynamics (QED) phenomena. These applications require precise control of cavity resonances mode. The cavity resonance is strongly depending on the lattice constant, a, and the air-hole radius, $r$, of photonic crystals. Slight modifications in the geometries of the photonic crystals will result in large differences in the dispersion characteristics. In this paper, by using BandSOLVE and FullWAVE software of RSoft Photonics CAD package, at first L1, L3 and L5 NCs in a semiconductor slab have been simulated in a hexagonal lattice of air-holes (radius $\mathrm{r}$ ) with lattice constant $\mathrm{a}=270 \mathrm{~nm}$ and constant fill factor $(\mathrm{r} / \mathrm{a}=0.29)$. Then, with radius reduction of the two ends air-holes (TEA-H), different confinement characteristics of photonic modes such as: numbers of confined modes, wavelengths, quality factors and two dimensional field profiles have been investigated. Calculations have shown that when radius of (TEA-H) in the linear NCs with $\mathrm{n}$ missing air-holes in a line, Ln, reaches to nearly zero value, transition in the structure to the $\mathrm{L}(\mathrm{n}+2) \mathrm{NCs}$, with roughly different qualities has been observed. Also, improvement in the quality factors of higher-order modes will be achieved. Understanding of the higher-order modes and two dimensional field profiles of the confined photonic modes are useful for the design of more efficient nano-lasers and observation of the cavity QED effects.
\end{abstract}

Keywords: Photonic Crystals, L1-L3-L5 Linear Nanocavities, Confined Modes, Field Profiles, Quality Factors

\section{Introduction}

A photonic crystal $(\mathrm{PhC})$ is a structure in which a periodic variation in the refractive index employed e.g. hexagonal lattice arrays of air-holes as a base, at the scale of the wavelength of light in one or more directions [1], as it depicted schematically in the Fig, 1. If the refractive index contrast of the arrays of air-holes and host dielectric/semiconductor material in the $\mathrm{PhC}$ is sufficiently large, it can result in the formation of a photonic band-gap (PBG) that means a range of frequencies in which the propagation of light is forbidden in the structure [1]. By introducing a defect in such periodic photonic crystals, it is possible to strongly localize light within a small volume [1-7]. Such defects are known as PhC nanocavities (NCs) and characterized by small modal volume $\left(\mathrm{V}_{\mathrm{m}}\right)$ and high quality factor, Q [3], making them attractive systems in which to study physical processes such as the Purcell effect which is characterized by the factor proportional to $\mathrm{Q} / \mathrm{V}_{\mathrm{m}}$ [7-9], exciton-photon strong coupling phenomena which is related to $\mathrm{Q} / \mathrm{V}_{\mathrm{m}}{ }^{1 / 2}$ [5], nonlinear optical effects which are characterized by $\mathrm{Q}^{2} / \mathrm{V}_{\mathrm{m}}[8,10]$, and ultra-low threshold nano-lasers $[1,2,6]$. Ideally to take full advantage of these physical effects, a three dimensional (3D) $\mathrm{PhC}$ is required $[1,11]$. However, fabricating such structures is technically challenging and frustrating process. Fabricating two dimensional (2D) $\mathrm{PhC}$ membrane (a dielectric slab membrane of high refractive index material with pattern e.g. a 2D triangular/hexagonal lattice arrays of air-holes - air columns - chemically etched/drilled) [12] is however much easier, making them an attractive alternative to $3 \mathrm{D} \mathrm{PhCs}$. In 2D PhC NCs in a semiconductor/dielectric slab, light is confined by a combination of in-plane PBG and vertical total-internal-reflection (TIR). Introducing a NC into a 2D $\mathrm{PhC}$ slab can be achieved in a couple of ways: by missing one or more air-holes (not etched) known as a donor-type $\mathrm{NC}[3,13,14]$, or by enlarging an air-hole, known as an accepter NC [13, 14]. In this paper, we computationally investigate some of the donor-type NCs, (the linear kind of $\mathrm{PhC} \mathrm{NCs})$. These linear $\mathrm{PhC} \mathrm{NCs}$, with " $\mathrm{n}$ " missing 
air-holes, "Ln", (such as L1, L3 and L5), are very useful in wavelength tuning of cavity modes, control of polarizations, conductivity and wave-guiding capability via lattice structure tuning and variety in designs. Based upon radius reduction in these $\mathrm{Ln} \mathrm{NCs}$, different confinement characteristics such as, number of confined modes, wavelength, quality factor and $2 \mathrm{D}$ field profiles of confined modes have been examined. When radius of two ends air-holes (TEA-H) in a linear Ln NCs, approximately reaches to zero, structural behavior transition to the $\mathrm{L}(\mathrm{n}+2)$ NCs, with different qualities would be possible to be observed.

This paper is separated into four sections: section 2 describes strategy for NCs simulations and amount of radius reduction of TEA-H in each step. Section 3 explains the procedure for calculation of $\mathrm{PBG}$ in $2 \mathrm{D} \mathrm{PhC} \mathrm{slab,}$ cavity Q factor, resonant wavelengths of $\mathrm{NC}$ modes, computational results and related outcomes. Finally, section 4 and 5 provide some discussions and conclusions.

\section{Simulations}

The L1, L3 and L5 NCs in a GaAs slab, (effective refractive index $\mathrm{n}_{\text {eff }}=3.09$ ), have been simulated in a triangular/hexagonal lattice of air-holes (radius $r$ and refractive index $\mathrm{n}=1$ ) with lattice constant $\mathrm{a}=270 \mathrm{~nm}$ and constant fill factor ratio, $\mathrm{r} / \mathrm{a}=0.29$. In the center of $\mathrm{NC}$, quantum dots have been considered as internal sources and then are excited with pulses for obtaining allowed resonant modes.

The L1 NC is the simplest kind of PhC linear NCs, Ln, which is created with omitting of one air-hole from etching, (creation of one point defect), in center of 2D PhC membrane. Also, L3 and L5 NCs, are designed with three and five missing air-holes respectively along a line, (creation of line defect), in the center of GaAs slab. Then, the radius size of two adjacent TEA-H, (existed in horizontal axis), can be reduced in several steps. The amount of air-hole reduction is considered e.g. $15.6 \mathrm{~nm}$ in each of five steps. In any step, wavelength and Q factor of the resonant modes, experiencing changes. Selection of numbers of steps and amount of radius reduction in each step is arbitrary. However, in radius less than $0.3 \mathrm{~nm}$, there is not any noticeable change in confinement characteristics. A schematic diagram of simulated L1, L3 and L5 NCs, and steps of radius reduction in the structures are shown in the Fig. 1.

\section{Calculations}

For calculating and plotting the band structure diagram, a typical 2D PhC slab structure is considered and shown in the Fig. 2-a. With attention to simulated slab structure, (air-holes patterned in a semiconductor slab), the PBG is formed for wavelengths with TE polarization, in range of 931-1179 nm. The confined modes in the L1, L3 and L5 NCs and modified ones, (L1, L3 and L5 NCs with reduced air-holes), existed in the PBG have been appeared with use of plan wave method (PWM) and finite different time domain (FDTD) method which are provided in the RSoft Photonics CAD package. By means of related softwares such as BandSOLVE and FullWAVE included in the package for realizing the PBG and confined modes, typical computational results are obtained and shown in the Fig. 2-a and 2-b respectively. Using such results we can deal with calculating resonance mode wavelength, $(\lambda)$, and cavity quality factor, $(\mathrm{Q})$ and discussions regarding confinement of individual modes.

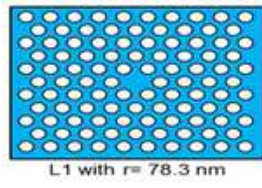

(a)

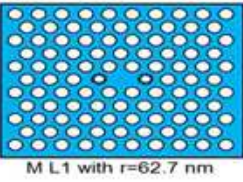

(b)

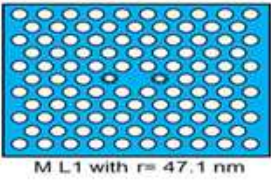

(c)

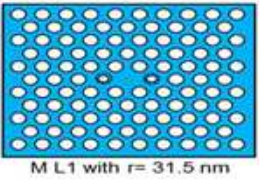

(d)

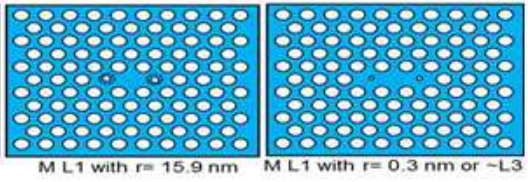

(e) (f)

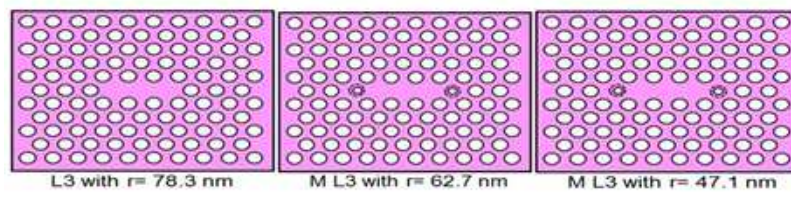

(g)

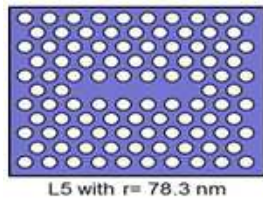

(m) (h)

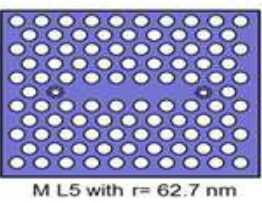

(n) (i)

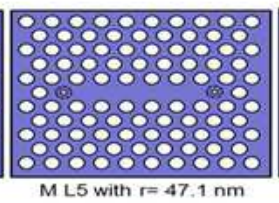

(o)

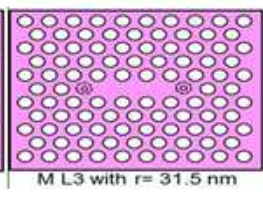

(j)

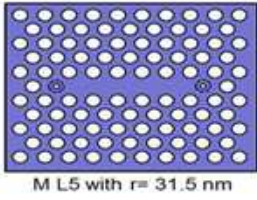

(p)

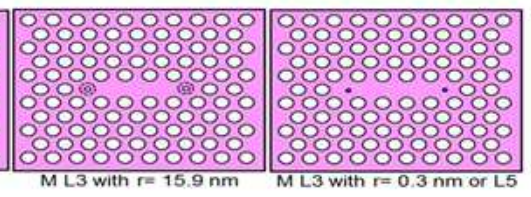

(k)

(I)

Figure 1. Schematic diagram of: $a-f) L 1, g-l)$ L3, m-r) L5 NCs and steps of two ends air-holes (TEA-H) radius reduction. 

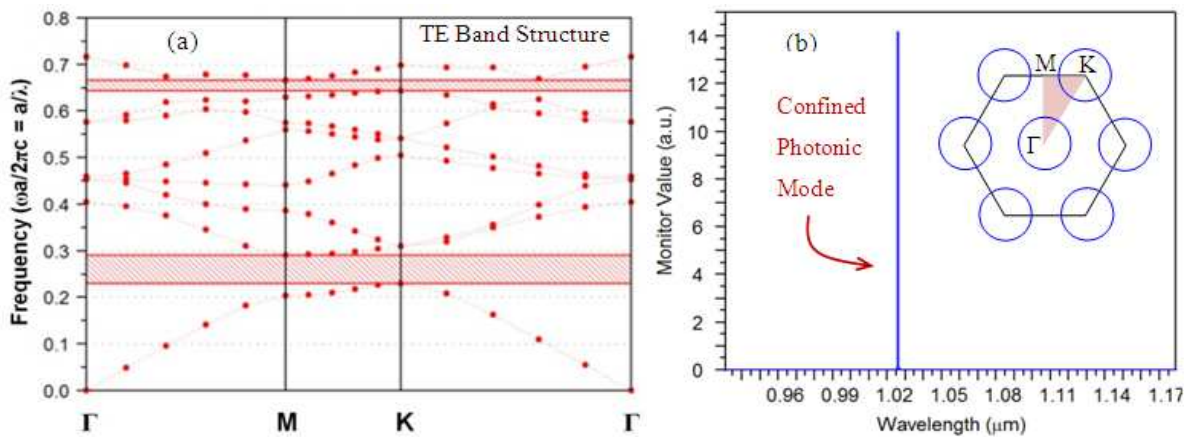

Figure 2. a) The photonic band structure and PBGs with TE mode polarization, for a simulated $2 D P h C$, and b) Computational spectrum of electromagnetic field from vertical component of confined mode; Inset: related Brillouin zone, with irreducible zone colored light pink.

A quantity which can show the strength of $\mathrm{NC}$ in resonant mode confinement is quality factor $(\mathrm{Q})$. The $\mathrm{Q}$ factor is expressed as follows:

$$
Q \equiv \omega_{0} \frac{U(t)}{-d U(t) / d t}
$$

Where, $\omega_{0}$ is the angular frequency of the cavity mode and $\mathrm{U}(\mathrm{t})$ is the total energy stored in the optical cavity. However, for calculation of $\mathrm{Q}$ with use of radiation spectrum, one can use the relation $\mathrm{Q}=\lambda / \Delta \lambda$; where $\lambda$ and $\Delta \lambda$ are wavelength and full width at half maximum (FWHM) of the mode respectively.

In each step of radius reduction, wavelength and $\mathrm{Q}$ factor of resonant modes would be altered. Thus, $\lambda \mathrm{s}$ and Qs in each step are calculated separately and summarized in the Table 1.

For better comparison and understanding of $\lambda \mathrm{s}$ and Qs variations, some typical related graphs for L1, L2 and L3 NCs were plotted and shown in the Fig. 3, Fig. 4 and Fig. 5 respectively.

Table 1. Wavelength and quality factor variations in different steps of simulation

\begin{tabular}{|c|c|c|c|c|c|}
\hline \multirow{2}{*}{$\begin{array}{c}\text { Type of } \\
\text { nanocavity }\end{array}$} & \multirow{2}{*}{$\begin{array}{l}\text { Radius of both ends } \\
\text { air-holes, } r \text { (nm) }\end{array}$} & \multicolumn{2}{|c|}{ Wavelength of resonant modes, $\lambda(\mathrm{nm})$} & \multicolumn{2}{|c|}{ Quality factor of resonant modes, $Q$} \\
\hline & & First mode & Second mode & First mode & Second mode \\
\hline \multirow{6}{*}{ L1 } & 78.3 & - & - & - & - \\
\hline & 62.7 & 940.1 & - & 1106 & - \\
\hline & 47.1 & 966.8 & - & 6906 & - \\
\hline & 31.5 & 996.5 & - & 7666 & - \\
\hline & 15.9 & 1081.9 & - & 5410 & - \\
\hline & 0.3 & 1026.0 & - & 10260 & - \\
\hline \multirow{6}{*}{$\mathbf{L 3}$} & 78.3 & 1026.0 & - & 10260 & - \\
\hline & 62.7 & 1045.7 & - & 5229 & - \\
\hline & 47.1 & 1064.5 & - & 10645 & - \\
\hline & 31.5 & 1081.6 & 940.9 & 5408 & 1001 \\
\hline & 15.9 & 1094.3 & 946.7 & 5472 & 2152 \\
\hline & 0.3 & 1098.4 & 948.8 & 5492 & 2497 \\
\hline \multirow{6}{*}{ L5 } & 78.3 & 1098.4 & 948.8 & 5492 & 552 \\
\hline & 62.7 & 1105.8 & 967.3 & 3686 & 4031 \\
\hline & 47.1 & 1112.7 & 986.9 & 11127 & 6168 \\
\hline & 31.5 & 1119.1 & 1004.7 & 11191 & 5024 \\
\hline & 15.9 & 1124.3 & 1017.4 & 1249 & 5087 \\
\hline & 0.3 & 1126.1 & 1021.3 & 5631 & 5107 \\
\hline
\end{tabular}
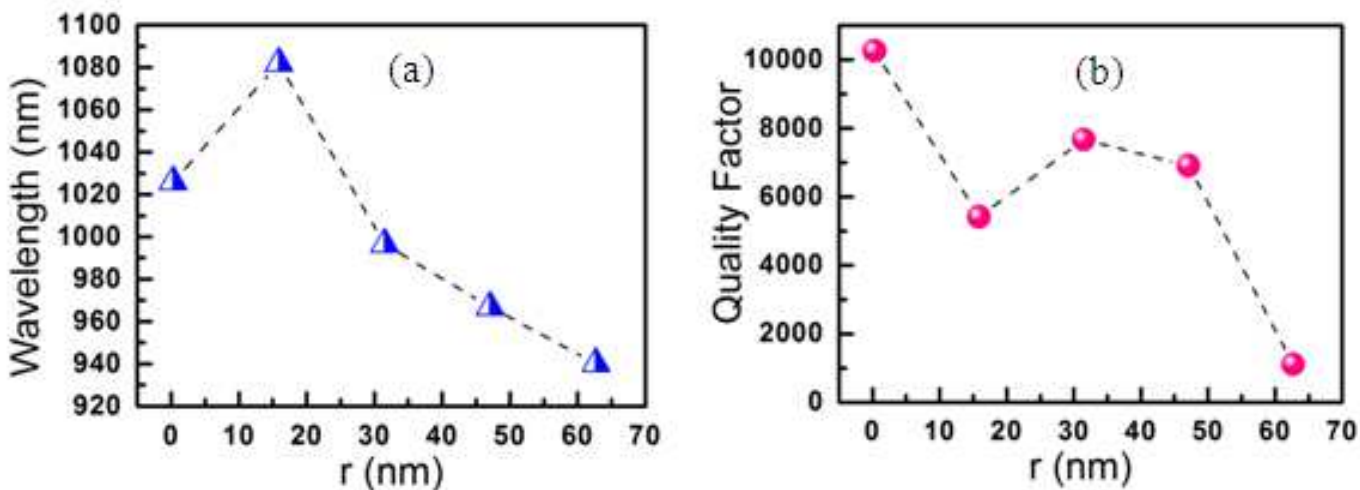

Figure 3. Variation of a) wavelength and b) quality factor of resonant cavity mode in $L 1 \mathrm{NC}$, in terms of radius reduction of (TEA-H), $r(n m)$. 

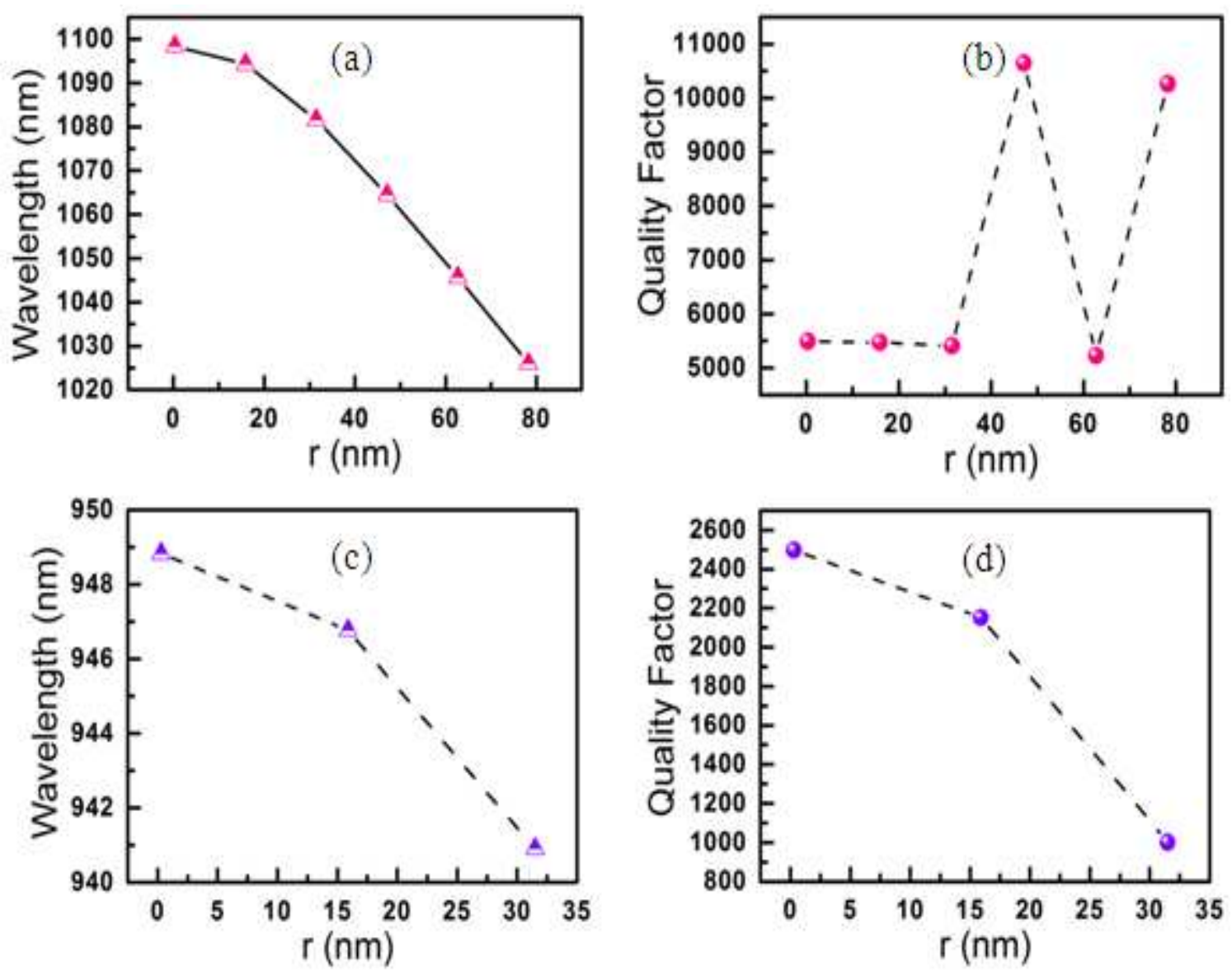

Figure 4. Variation of wavelength and quality factor of a) and b) first resonant mode, c) and d) second resonant mode in the L3 NC, in terms of radius reduction of (TEA-H), $r(\mathrm{~nm})$.

To have better knowledge regarding field distributions in the cavity, 2D patterns of cavity mode related to the vertical component of electromagnetic field in a modified L1 (ML1) with decreased radius, and normal L3 NC, have been plotted and shown in the Fig. 6.

It is clearly observable that the $2 \mathrm{D}$ patterns of the field of resonant cavity mode in different steps of simulation $(a, b$, $\mathrm{c}$ and $\mathrm{d}$ in the Fig. 3) have been changing slowly, and finally in last step ML1 NC with $\mathrm{r}=0.3 \mathrm{~nm}$, have exactly resonant cavity mode pattern which is computed in the L3 $\mathrm{NC}$, as somehow we could expected (similarity between parts e and $\mathrm{f}$ in the Fig. 3). As radius of TEA-H is decreased, modal volume of the cavity were increased and therefore, peak intensity distribution of cavity mode could be concentrated further than cavity walls, and then in final stage, it has redistributed in the center of NC with high localization. The benefit of this phenomenon at low dimensions is lower loss or higher quality factor.

\section{Discussions}

As we can see in the Fig. 3, with radius reduction of TEA-H in the L1 NC, when structure parameter variations reach to appropriate conditions, fundamental confined mode appears, and by continuing further reduction of the radius with uniform procedure, mode's wavelength and $\mathrm{Q}$ factor, increased approximately in a uniform rate. Calculations have shown that in the last step of radius reduction, where radius size of two air-holes is $0.3 \mathrm{~nm}$ (nearly zero), wavelength and Q factor of the confined mode are $1026 \mathrm{~nm}$ and roughly 10260 respectively (row 6 in the Table 1). These values are in agreements with the ones of first confined mode in the L3 NC (row 7 in the Table 1). In fact, structure transition to the $\mathrm{L} 3 \mathrm{NC}$ is observable. Also, the $2 \mathrm{D}$ field patterns of the cavity mode in the two mentioned $\mathrm{NC}$ are similar (Fig. 6- e and f).

In the early steps of TEA-H radius reduction in the $\mathrm{L} 3 \mathrm{NC}$, only variations in characteristics of the first confined mode such as wavelength's increasing and Q factor's fluctuations have been seen (Fig. 4- a and b). But, from step three (where radius size of TEA-H reaches to $31.5 \mathrm{~nm}$ ) up to end, another mode which we called second mode, has appeared in the range of the PBG. Also, variations in wavelength and $\mathrm{Q}$ factor of this mode have seen in increasing manner (Fig. 4- c and d). However, in the last three steps, Q factor of both modes has shown very slight change (Fig. 4- $b$ and $d$ ).

The L5 NC has two confined resonant modes with wavelengths 948.82 and $1098.4 \mathrm{~nm}$ in the PBG (row 13 in the Table 1). These values are as the same as the ones in L3 $\mathrm{NC}$ with reduced TEA-H radius to nearly zero (row 12 in the Table 1). However, only $Q$ factor of the first mode with higher wavelength in the two mentioned structures has 
compatible transition with the same Q value (5492). The Q factors of the second mode in these structures have different values ( 2497 for the $\mathrm{L} 3 \mathrm{NC}$ with reduced radius $0.3 \mathrm{~nm}$ and 552 for L5 NC). Difference in the Q factors of the second mode in these two structures is due to alterations in the concentration of mode intensity across the NC and lowering
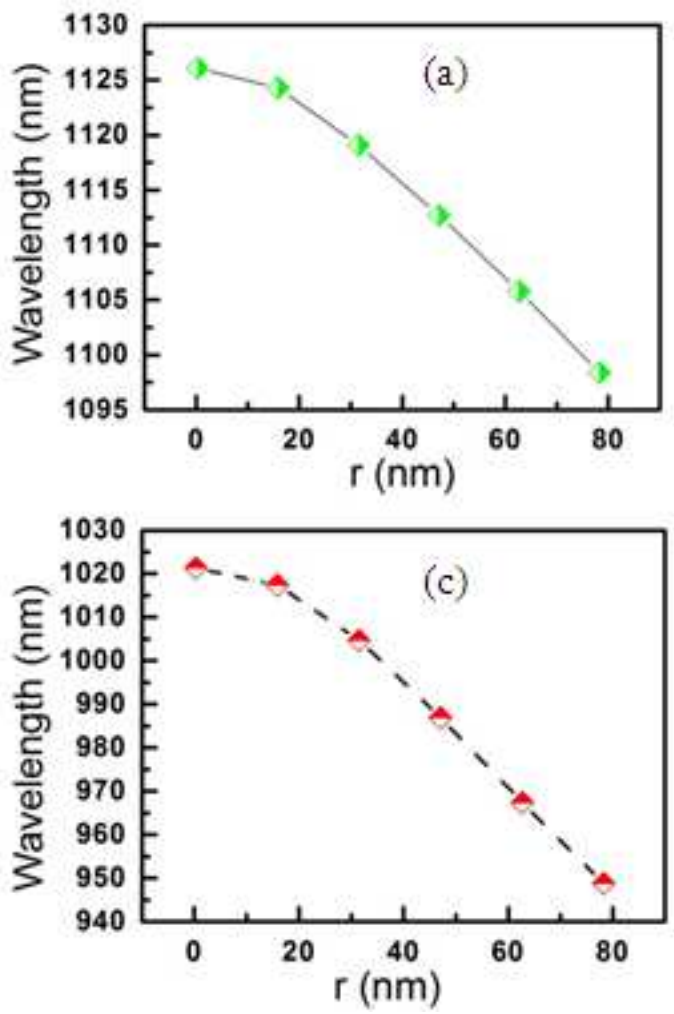

loss of energy by intensifying modes peaks away from sidewalls via mechanism based upon controlling cavity shape. In the former structure transition, from L3 NC with TEA-H radius $0.3 \mathrm{~nm}$ to $\mathrm{L} 5 \mathrm{NC}$, less variation in comparison with previous transition are seen due to initial larger cavity size.
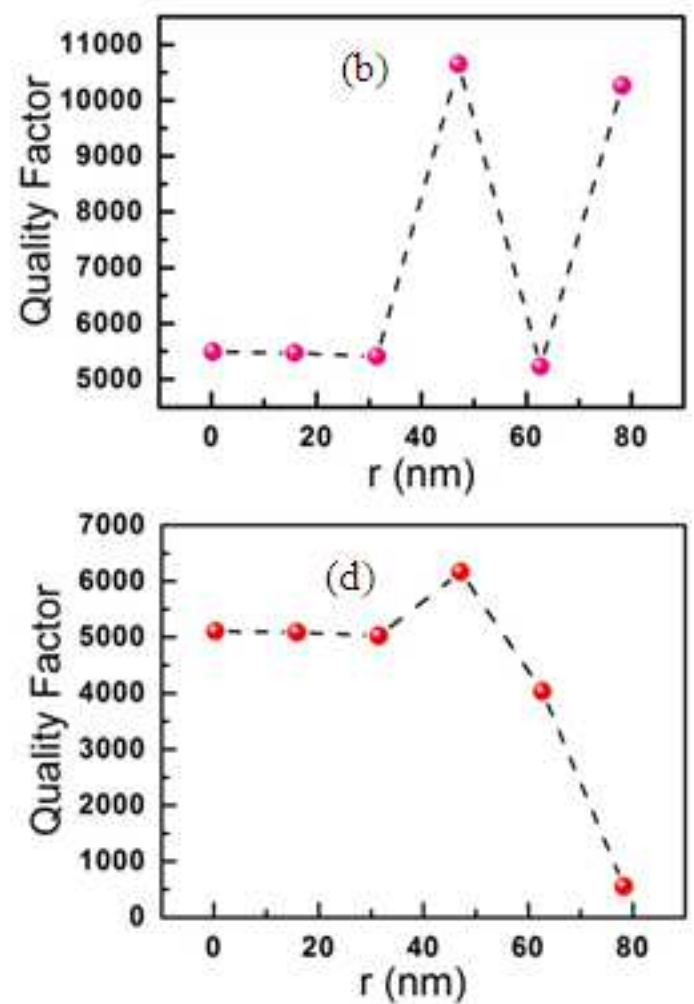

Figure 5. Variation of wavelength and quality factor of a) and b) first resonant mode, c) and d) second resonant mode in the L5 NC, in terms of radius reduction of (TEA-H), $r$ (nm)

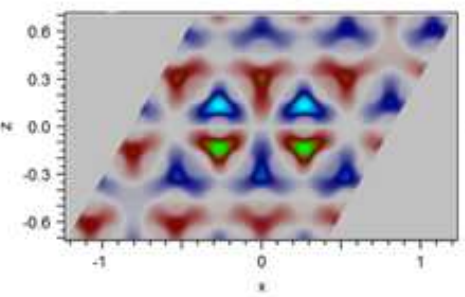

(a)

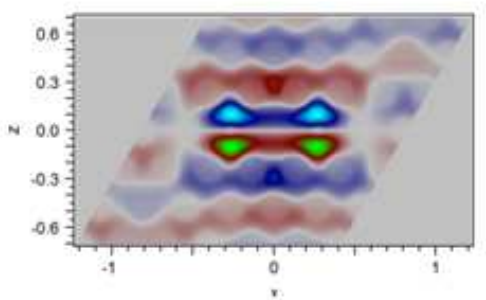

(d)

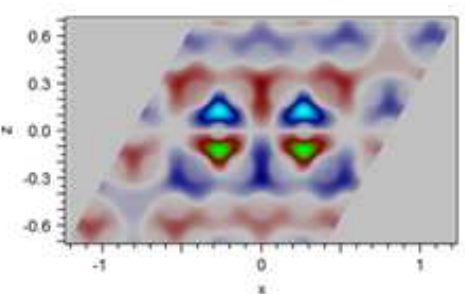

(b)

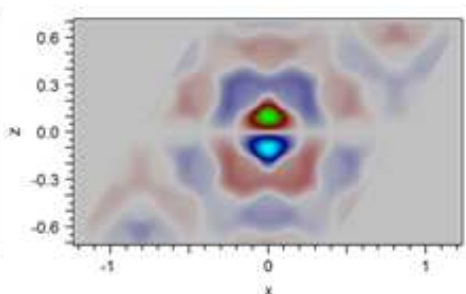

(e)

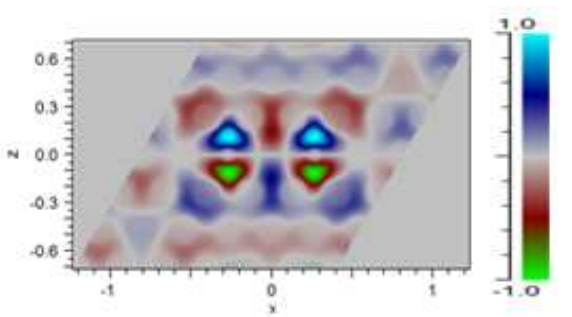

(c)

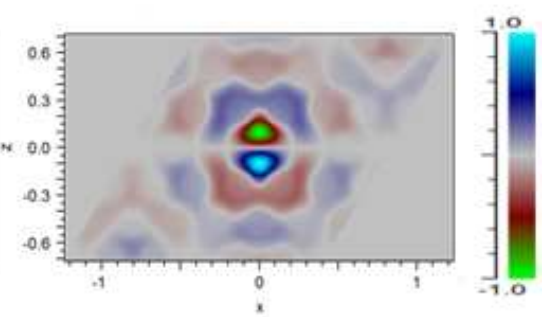

(f)

Figure 6. The 2D patterns of cavity mode related to the vertical component of electromagnetic field in a modified L1 (ML1) with decreased radius to a) $r=62.7 \mathrm{~nm}, b) r=47.1 \mathrm{~nm}, c) r=31.5 \mathrm{~nm}, d) r=15.9 \mathrm{~nm}, \mathrm{e}) r=0.3 \mathrm{~nm}$ (nearly zero), and f) the normal L3 NC without any modification.

Furthermore, reducing radius of TEA-H in L5 NC, we can see the wavelengths of both confined modes, have almost increased uniformly (Fig. 5- a and c), and in the last step have reached to 1126.1 and $1021.3 \mathrm{~nm}$. These values are in agreement with wavelengths of confined modes in the L7 $\mathrm{NC}$, (which is simulated in the same way as for the L3 and L5 NCs). However, the Q factors of two confined modes are different. The $\mathrm{Q}$ factors of resonant modes in the $\mathrm{L} 5 \mathrm{NC}$ with 
TEA-H radius $0.3 \mathrm{~nm}$ are 5631 and 5107 which are not in compatibility with 1408 and 2042 (calculated for the confined modes in the exact L7 NC). Trend of changes in the quality factor of L5 NC modes are to some extent irregular (Fig. 5- b and d). Nevertheless, it should be noted that the quality factor of the second confined mode, in second phase of radius reduction, has reached to more than eleven times of the initial amount. Indeed, in transition structure from L5 $\mathrm{NC}$ with radius of TEA-H reduced to zero, to make $\mathrm{L} 7 \mathrm{NC}$, only wavelengths of confined modes have similarity and good compatibility, without any further overall quality factor improvements. In other words, in larger Ln NCs, as the longer axis size is increased, qualities of structural transitions between two consecutive structures would not be in any further beneficial.

\section{Conclusions}

Confined photonic modes in the variety of linear NCs designs could be controlled with NC's TEA-H radius reduction. In this paper, wavelength and quality factor variations and field pattern were investigated computationally. The L1 NC appeared to have high Q value mode as high as 10260 and showed good structural transition to the $\mathrm{L} 3 \mathrm{NC}$ with reducing two adjacent air-holes of single missing air-hole. In the last step of TEA-H radius reduction, structural transition from L1 to L3 $\mathrm{NC}$ with a good compatibility between wavelengths and $\mathrm{Q}$ factors of the fundamental mode were observed. In the $\mathrm{L} 3 \mathrm{NC}$, with TEA-H radius reduction, second confined mode was appeared in the PBG region. Transition of structure from the L3 NC to L5 and then L7 NC were investigated as well, and shown good wavelength transition. However, some discrepancies were found in view of Q factors of the second mode in transition structure from L5 NC to L7 NC. In spite of this matter, $\mathrm{Q}$ factor of the second photonic mode in the L5 NC was reached to more than eleven times of the initial value. Understanding of the higher-order modes is useful for the design of more efficient nano-lasers and for the observation of cavity quantum electrodynamics effects. Also, 2D field profiles of the confined modes gives idea for the estimation values of the cavity modal volumes which are helpful for the Purcell factor calculation.

\section{References}

[1] J. D. Joannopoulos, S. G. Johnson, J. N. Winn, R. D. Meade, "Photonic Crystals - Molding the Flow of Light", Princeton University Press, Princeton, New Jersey, 2nd ed, (2008).

[2] K. Sakada, "Optical Properties of Photonic Crystals", Springer, 2nd ed, (2004).

[3] Y. Akahane, T. Asano, B. S. Song, S. Noda, "High-Q photonic nanocavity in two-dimensional photonic crystal", Nature 425, 944 (2003).

[4] K. J. Vahala, "Optical Microcavities", Nature 424, 839 (2003).

[5] T. Yoshie, A. Scherer, J. Hendrickson, G. Khitrova, H. M. Gibbs, G. Rupper, C. Ell, O. B. Shchekin, D. G. Deppe, "Vacuum Rabi splitting with a single quantum dot in a photonic crystal nanocavity", Nature 432, 200 (2004).

[6] S. Strauf, K. Hennessy, M. T. Rakher, Y. S. Choi, A. Badolato, L. C. Andreani, E. L. Hu, P. M. Petroff, and D. Bouwmeester, "Self-tuned quantum dot gain in photonic crystal lasers", Phys. Rev. Lett. 96, 127104 (2006).

[7] W-H. Chang, W-Y. Chen, H-S. Chang, T-P. Hsieh, J-I. Chyi, and T-M. Hsu, "Efficient single-photon sources based on low-density quantum dots in photonic-crystal nanocavities", Phys. Rev. Lett. 96, 117401 (2006).

[8] M. Notomi, A. Shinya, S. Mitsugi, G. Kira, E. Kuramochi, and T. Tanabe, "Optical bistable switching action of $\mathrm{Si}$ high-Q photonic-crystal nanocavities", Optics Express 13, 2678 (2005).

[9] E. M. Purcell, "Spontaneous emission probabilities at radio frequencies”, Phys. Rev. 69, 681 (1946).

[10] D. Englund, I. Fushman and J. Vučković, "General recipe for designing photonic crystal cavities”, Optics Express 13, 5961 (2005).

[11] S. Ogawa, M. Imada, S. Yoshimoto, M. Okano, S. Noda, "Control of Light Emission by 3D Photonic Crystals", Science 305, 227 (2004).

[12] T. F. Krauss, R. M. De La Rue, S. Brand, "Two-dimensional photonic-bandgap structures operating at near-infrared wavelengths", Nature 383, 699 (1996).

[13] Y. Akahane, T. Asano, B. S. Song, and S. Noda, "Investigation of high-Q channel drop filters using donor-type defects in two-dimensional photonic crystal slabs", Appl. Phys. Lett. 83, 1512 (2003).

[14] S. Noda, A. Chutinan, M. Imada, "Trapping and emission of photons by a single defect in a photonic bandgap structure", Nature 407, 608 (2000). 